American Chemical Society, Langmuir, La0701948g, Supporting Information Page S1

\title{
Periodic Mesoporous Organosilicas with Multiple Bridging Groups and Spherical Morphology
}

\section{Eun-Bum Cho, ${ }^{\dagger}$ Dukjoon Kim, ${ }^{\dagger *}$ Mietek Jaroniec $^{\ddagger *}$}

${ }^{\dagger}$ Polymer Technology Institute, Department of Chemical Engineering, Sungkyunkwan University, Suwon, Gyeonggi-do 440-746, Korea

${ }^{\ddagger}$ Department of Chemistry, Kent State University, Kent, Ohio, 44240 USA

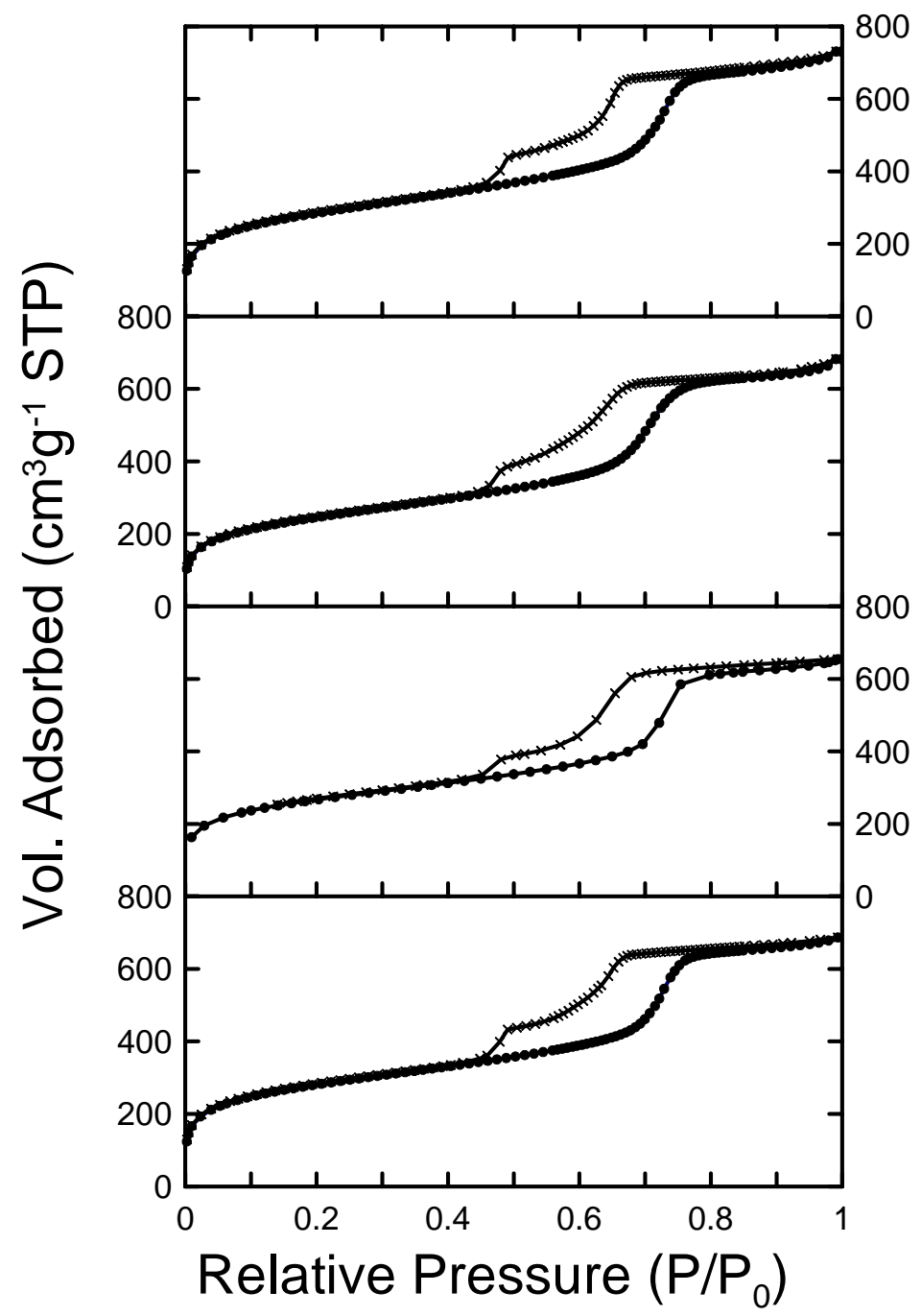

Figure S1. Nitrogen adsorption-desorption isotherms for the selected PMOs with phenylene, thiophene, and ethane bridging groups; (a) PE11, (b) TE11, (c) PT11, and (d) PTE111. 
American Chemical Society, Langmuir, La0701948g, Supporting Information Page S2
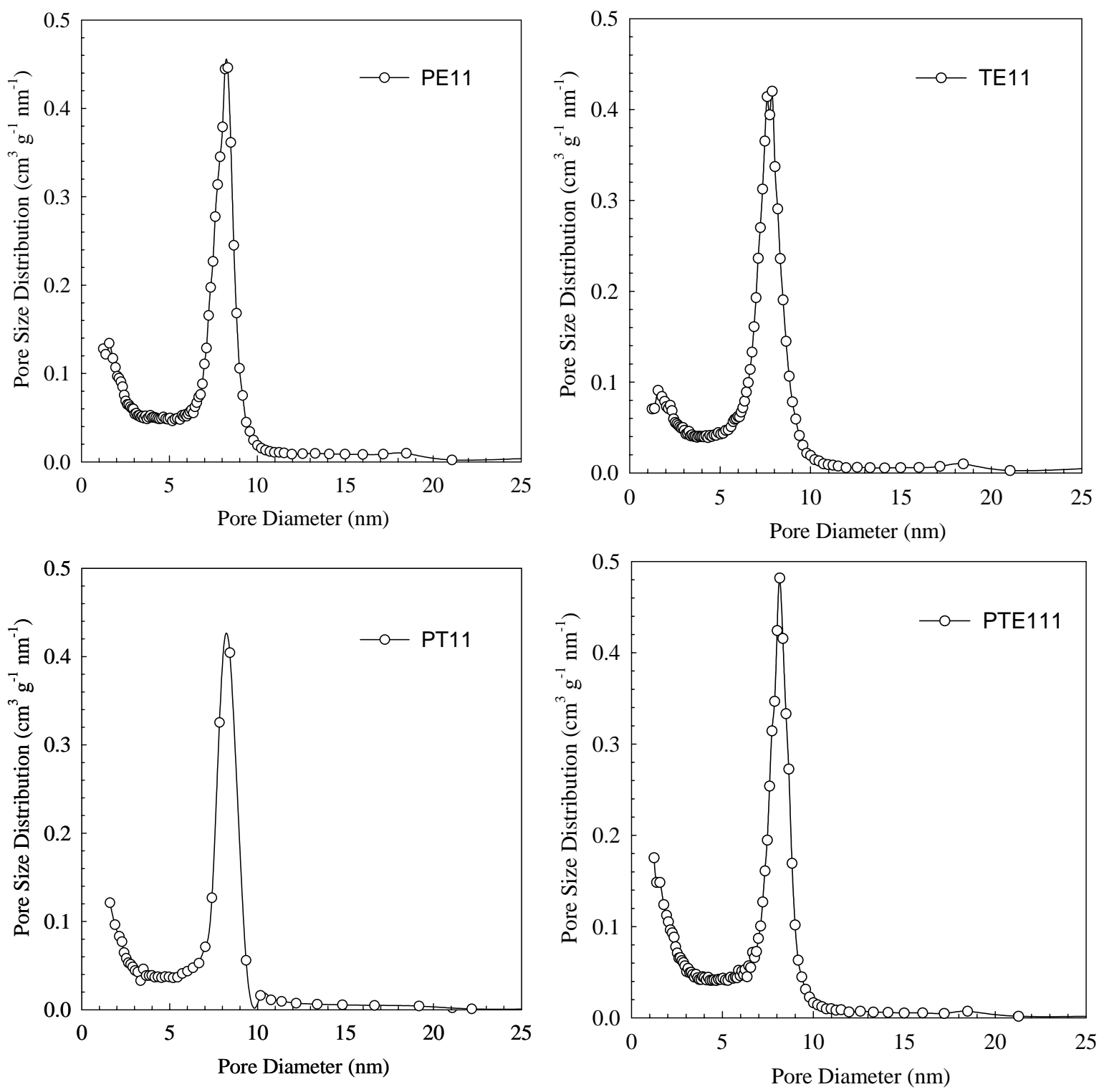

Figure S2. Pore size distributions obtained by the improved KJS method for the selected PMOs with phenylene, thiophene, and ethane bridging groups (PE11, TE11, PT11 and PTE111). 
American Chemical Society, Langmuir, La0701948g, Supporting Information Page S3

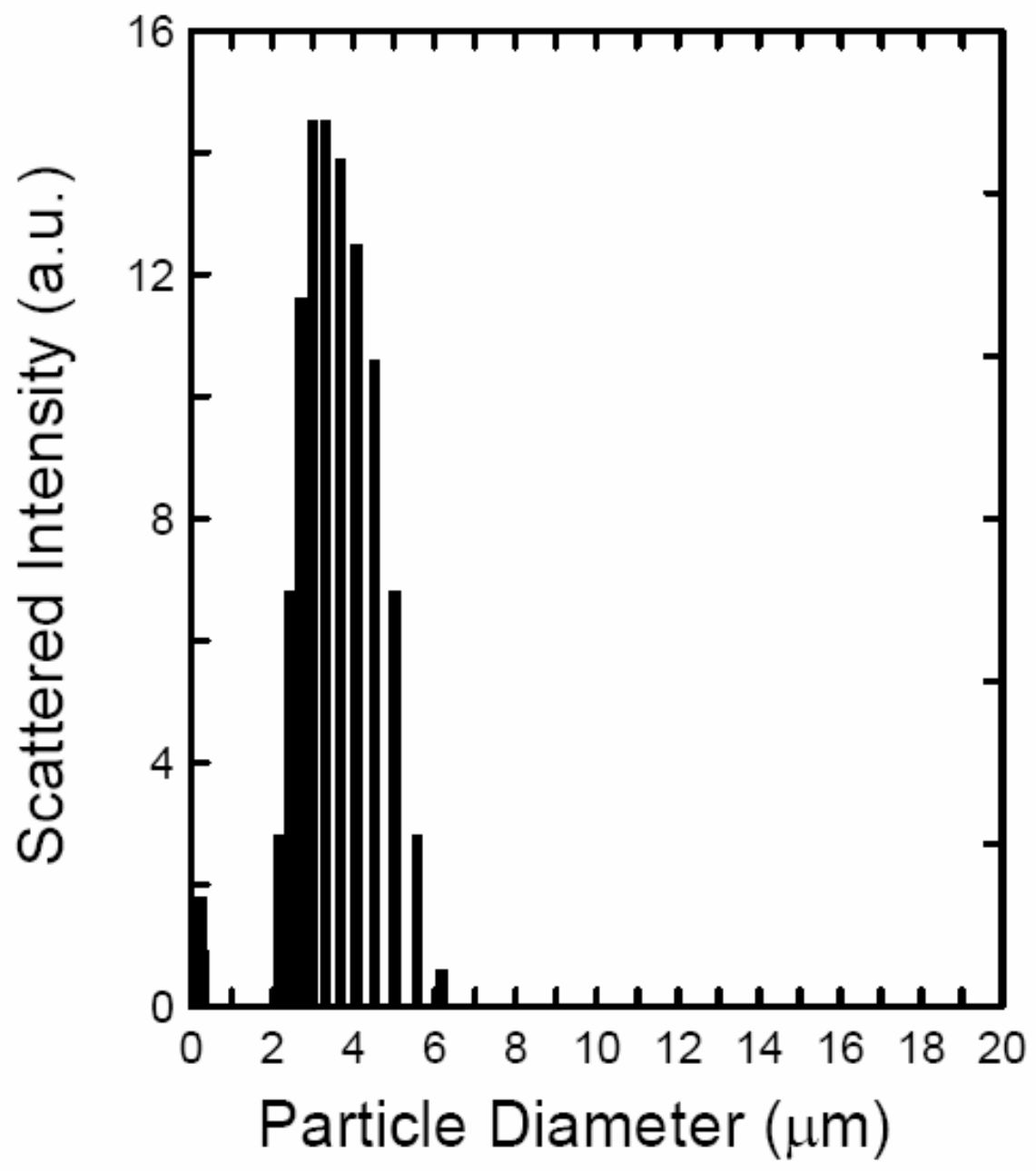

Figure S3. Particle size distribution obtained form the electrophoretic light scattering data for the phenylene-ethane PMO (PE11); the average particle size is about $2.9 \mu \mathrm{m}$. 
American Chemical Society, Langmuir, La0701948g, Supporting Information Page S4

(a)

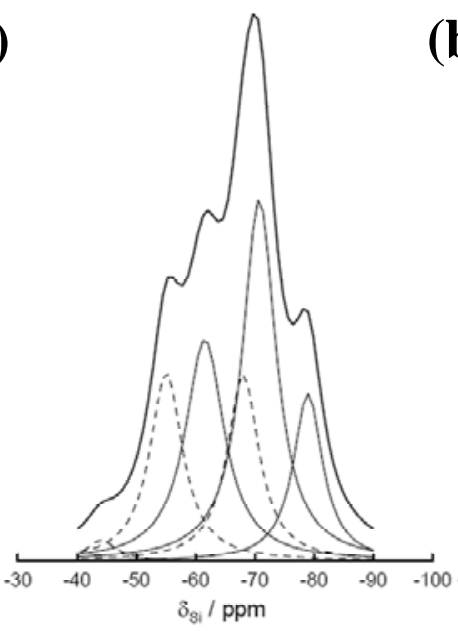

(d)

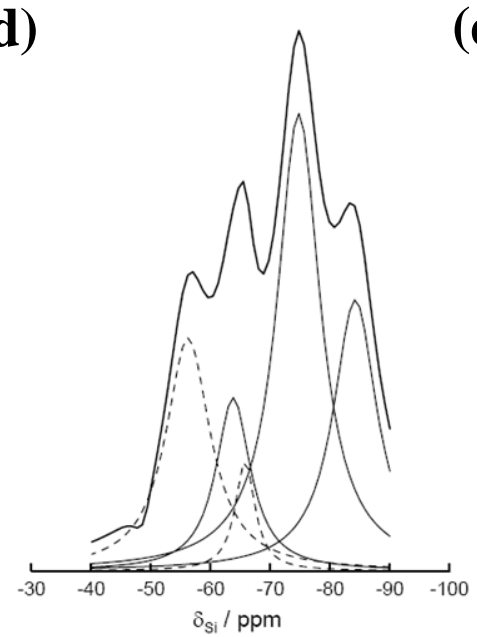

(g)

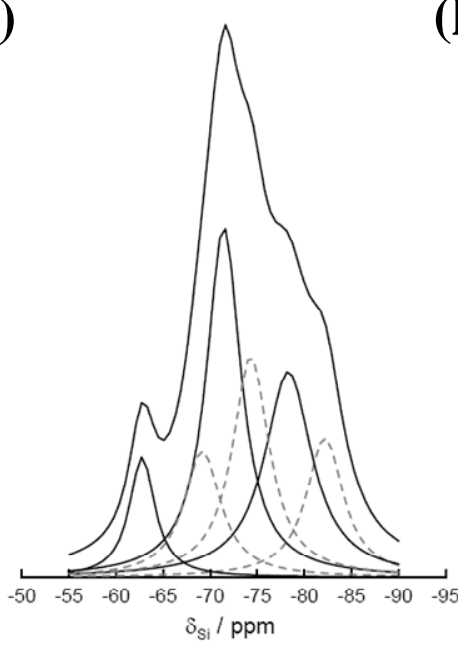

(b)

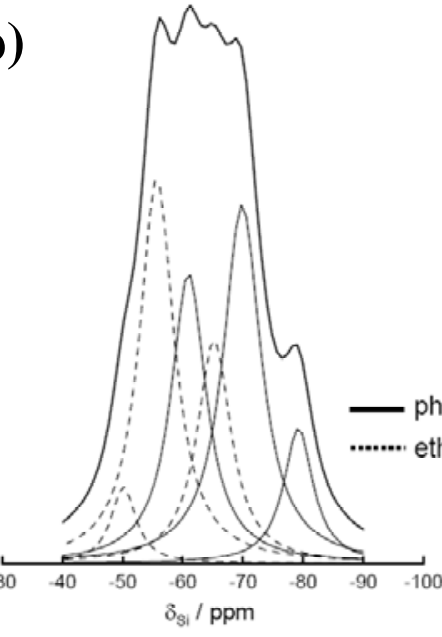

(e)
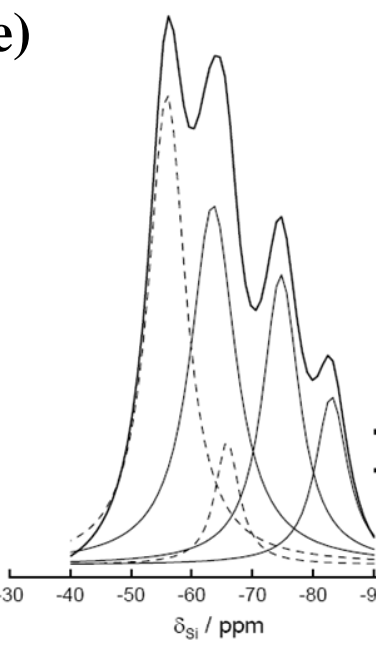

(h)

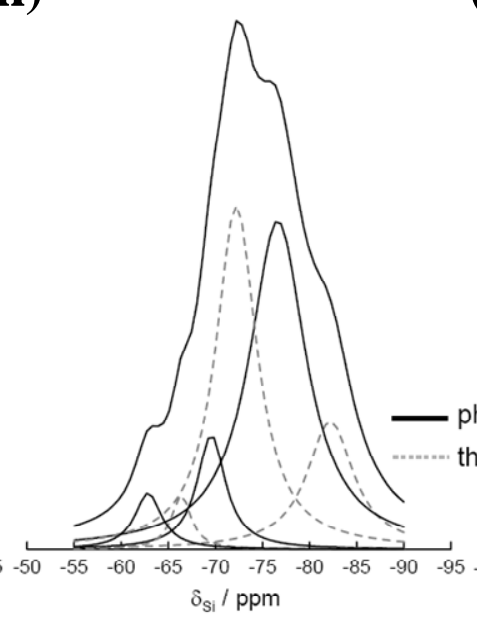

(c)

(f) 
American Chemical Society, Langmuir, La0701948g, Supporting Information Page S5
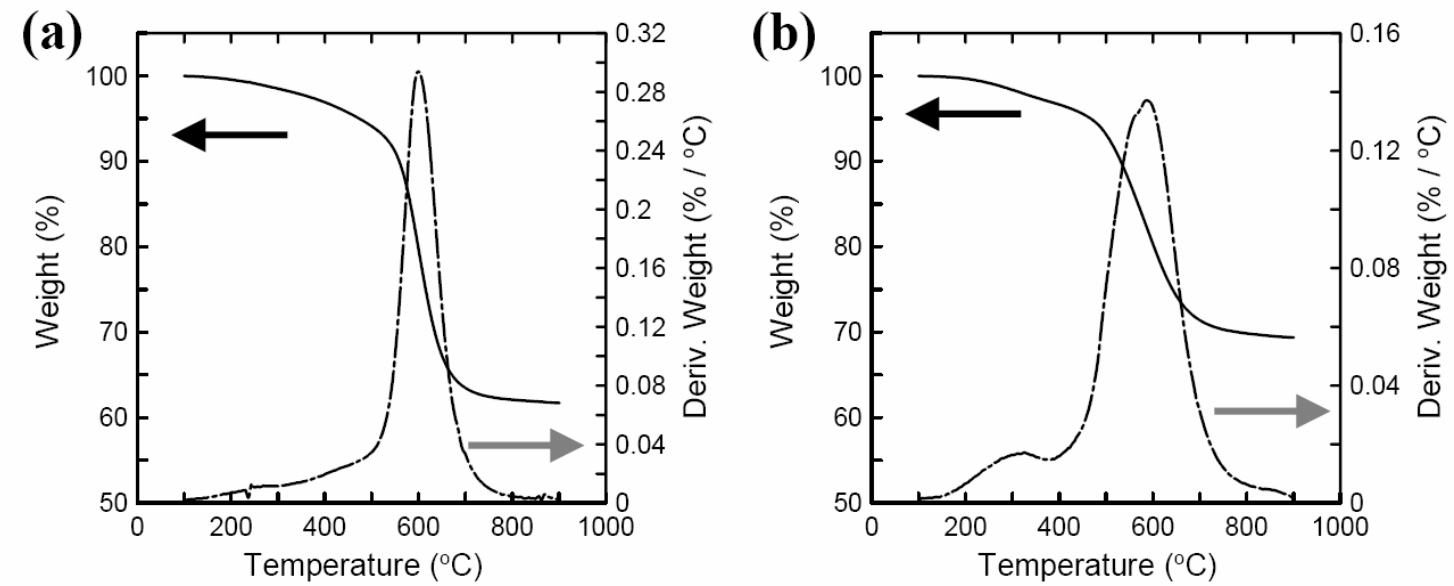

Figure S5. Thermogravimetric profiles recorded in flowing air for two samples of PMOs with phenylene, thiophene, and ethane bridging groups: (a) PT11 and (b) PTE111. 